\title{
Wer zahlt gewinnt
}

Michael Joswig

„Fußball ist keine Mathematik“. Diesen Satz musste sich der damalige Bayern-Trainer und vormalige Mathematiklehrer Ottmar Hitzfeld von seinem Vorstandschef KarlHeinz Rummenigge nach einem unglücklichen Unentschieden im Herbst 2007 anhören. Hat Rummenigge recht? Zu jeder Bundesligasaison fragen sich Experten und Fans aufs Neue, was denn am Ende den Meister eigentlich ausmacht. Die eine Mannschaft hat einen starken Sturm, die nächste verteidigt geschickt, und die dritte hat einen international erfahrenen Trainer. Spätestens seit der Weltmeisterschafts-Euphorie schießen überall die Tippgemeinschaften und Expertenrunden aus dem Boden. Kann man sich mit etwas Mathematik einen Vorteil bei der Bundesligawette verschaffen?

Fußball ist ein Fest für die Statistik. Alles wird gemessen und gezählt. Wer wann wieviele Tore geschossen hat sowieso, aber eben auch, wann welche Ecke geschossen wurde, wie viele Torschüsse links vorbei gingen und wie viele rechts, welcher Spieler welche Lufstrecke zurücklegt und wie lang er kein Tor geschossen hat. Im Zeitalter des Internets haben sich zahlreiche spezialisierte Webseiten dem Fußball verschrieben. Zum Beispiel www. rsssf.com ist eine echte Schatzgrube. Wer wissen möchte, welcher Club in der englischen Southern League (Ist Division) die Saison 1894/95 gewonnen hat (Antwort: Luton Town), ist hier genau richtig. Für die Frage nach der Zuschauerentwicklung speziell im deutschen Fußball ist man auf www.dsfs.de bestens aufgehoben. Daten ganz besonderer Art aber werden unter transfermarkt.de vorgehalten. Denn hier geht es um das Geld. Jeder Spielertransfer wird verzeichnet zusammen mit vielen Details und Gerüchten. Ob Ivan Rakitic vom FC Schalke 04 zu luventus Turin wechselt oder nicht, auf transfermarkt.de kann man es vermutlich zuerst erfahren. Vor allem aber bekommt jeder Spieler durch die Transfersummen einen numerischen Wert zugeordnet, der irgendwie seine Leistung auf dem Rasen reflektieren soll. Kann man aus diesen Informationen Hinweise auf das Abschneiden einer ganzen Mannschaft ziehen?

\section{Die Bundesligasaison 2007/08}

Am Ende der Saison 2007/08 stand wieder einmal der FC Bayern München als Meister fest, dicht gefolgt von Mannschaften, die auch in den Vorjahren stets auf den vorderen Rängen zu finden waren. Die Frage ist, inwieweit man das auch im Voraus hätte ahnen können. Hierzu lohnt es sich die Daten von transfermarkt.de vor Beginn der Saison heranzuziehen. In der Tabelle I sind die I8 Mannschaften der I. Bundesliga absteigend geordnet nach dem durchschnittlichen Transferwert ihrer Spieler (angegeben in Tausend Euro), also der monetären Entsprechung der in der Sportschau oft thematisierten "individuellen Klasse”. Zusätzlich sind die Gesamtgröße des

Bundesliga 2007/08. Wert: Stand 10. Juli 2007, Platz: Saisonende. (Quelle: transfermarkt.de)

\begin{tabular}{lcccrrrr}
\hline Verein & Kader & $\varnothing$-Alter & Marktwert in TEuro & $\varnothing$-Mw. & Rang & Platz & Differenz \\
\hline FC Bayern München & 27 & 26,2 & 233650 & 8654 & 1 & 1 & 0 \\
Werder Bremen & 33 & 24,2 & 134400 & 4073 & 2 & 2 & 0 \\
FC Schalke 04 & 28 & $25, I$ & 104500 & 3732 & 3 & 3 & 0 \\
VfB Stuttgart & 30 & 24,8 & 103350 & 3445 & 4 & 6 & -2 \\
Hamburger SV & 29 & 24,4 & 93650 & 3229 & 5 & 4 & 1 \\
Borussia Dortmund & 26 & 26,3 & 83200 & 3200 & 6 & 13 & -7 \\
Bayer 04 Leverkusen & 24 & 24,9 & 67625 & 2818 & 7 & 7 & 0 \\
Hertha BSC & 24 & 25,4 & 54300 & 2262 & 8 & 10 & -2 \\
I. FC Nürnberg & 32 & 25,0 & 70750 & 2211 & 9 & 16 & -7 \\
Eintracht Frankfurt & 25 & 25,9 & 50450 & 2018 & 10 & 9 & 1 \\
VfL Wolfsburg & 29 & 25,6 & 54300 & 1872 & 11 & 5 & 6 \\
Hannover 96 & 30 & 27,3 & 52650 & 1755 & 12 & 8 & 4 \\
VfL Bochum & 27 & 26,3 & 32850 & 1217 & 13 & 12 & 1 \\
Arminia Bielefeld & 31 & 26,6 & 35850 & 1156 & 14 & 15 & -1 \\
Karlsruher SC & 23 & 25,6 & 26300 & 1143 & 15 & 11 & 4 \\
MSV Duisburg & 31 & 26,6 & 27400 & 884 & 16 & 18 & -2 \\
FC Energie Cottbus & 30 & 26,4 & 22250 & 742 & 17 & 14 & 3 \\
Hansa Rostock & 25 & 25,5 & 18325 & 733 & 18 & 17 & 1 \\
\hline Gesamt & 504 & 25,7 & 1265800 & 2512 & & & \\
\hline
\end{tabular}




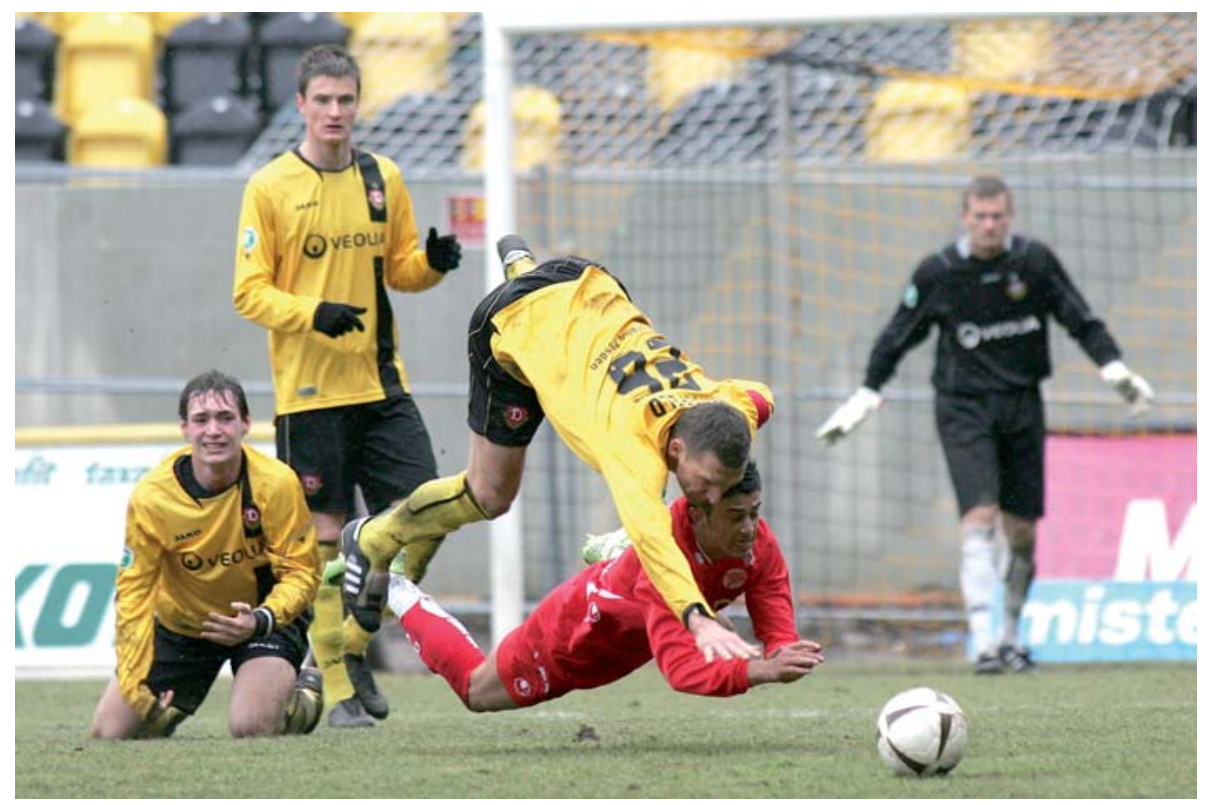

„Fußball ist keine Mathematik“ (Quelle: www.afb-media.de)

Kaders, das Durchschnittsalter und der Gesamtwert der Mannschaft aufgeführt. Die letzten beiden Spalten zeigen die Platzierung am Ende der Saison und die Differenz zwischen Durchschnittswertrang und Platzierungsrang. Es fällt unmittelbar auf, dass die Differenzen zumeist sehr gering sind. Als ein weiteres interessantes Detail sei angefügt, dass bei den beiden Mannschaften, die zwischen Investitionen und Erfolg das größte Missverhältnis ausweisen, Dortmund und Nürnberg, gegen Ende der Saison die Trainer gefeuert wurden.

\section{Rang-Korrelationen}

Wir wollen die Tabelle I mit den Methoden der elementaren Statistik untersuchen. Der Korrelationskoeffizient zweier Stichproben $x$ und $y$ (nach Pearson) berechnet sich zu

$$
r_{x, y}=\frac{s_{x, y}}{s_{x} s_{y}}
$$

mit

$$
s_{x, y}=\frac{1}{n-1} \sum_{i=0}^{n}\left(x_{i}-\bar{x}\right)\left(y_{i}-\bar{y}\right)
$$

und $s_{x}=\sqrt{s_{x, x}}$. Hier sind $\bar{x}$ und $\bar{y}$ die Mittelwerte der beiden Stichproben, und $n$ ist die Größe beider Stichproben. Es gilt stets $-1 \leq r_{x, y} \leq 1$. Im Allgemeinen sind die Stichproben reellwertig; es macht aber auch Sinn die Berechnung der Korrelation auf Ordinalzahlen anzuwenden, die von Reihenfolgen herrühren. In diesem Fall spricht man von Rang-Korrelation. In unserem Fall könnte $x_{i}$ der Spalte "Rang" der Tabelle I entsprechen und $y_{i}$ dem Wert in der Spalte "Platz". In der Tabelle 2 sind die Rang-Korrelationen der verschiedenen Merkmale aus Tabelle I mit dem Abschneiden am Ende der Saison aufgeführt. Die höchste Korrelation zum Erfolg besteht für den durchschnittlichen Wert der einzelnen Spieler.

Es bleibt zu klären, wie signifikant die berechneten Korrelationen sind. Ein naheliegendes Verfahren besteht darin, die berechnete Korrelation mit der Korrelation einer zufälligen Permutation zu vergleichen. Wollte man für das exakte Ergebnis alle I8!=6402 373705728000 Permutationen testen, so würde das auch auf einem schnellen Rechner ziemlich lange dauern. Daher begnügt man sich in der Praxis oft mit einem approximativen Permutationstest. Hierfür zieht man eine gewisse Anzahl von Permutationen zufällig und zählt, welcher Anteil unter diesen eine höchstens ebenso große Korrelation aufweist wie die auf Signifikanz zu testende Stichprobe. Die aus 100000 zufälligen Permutationen geschätzten Signifikanzwerte sind ebenfalls der Tabelle 2 zu entnehmen.

Die Signifikanz der Korrelation zwischen der Platzierung am Ende der Saison und dem durchschnittlichen Transferwert der Spieler ist mit über 99,99\% überwältigend, dicht gefolgt von der Signifikanz der Korrelation zum Gesamtwert der Mannschaft. Es besteht aber auch eine fast ebenso starke Signifikanz der Korrelation zum Alter der Spieler (je jünger desto besser). Dagegen schien 2007/08 die Größe des Kaders gar keinen Einfluss zu haben.

\begin{tabular}{|c|c|c|c|c|}
\hline & Kader & $\varnothing$-Alter & Marktwert & $\varnothing-M w$. \\
\hline Korrelation & $-0,04438$ & 0,47988 & 0,76058 & 0,80186 \\
\hline Signifikanz & 0,43122 & 0,97767 & 0,99980 & 0,99997 \\
\hline
\end{tabular}

Bundesliga 2007/08 - Korrelation zur Platzierung am Saisonende 
Bundesliga 2008/09. Wert: Stand II. August 2008, Platz: nach 17 Spieltagen. (Quelle: transfermarkt.de)

\begin{tabular}{|c|c|c|c|c|c|c|c|}
\hline Verein & Kader & $\varnothing$-Alter & Marktwert in TEuro & $\varnothing-M w$. & Rang & Platz & Differenz \\
\hline FC Bayern München & 23 & 26,3 & 252900 & 10996 & I & 2 & -1 \\
\hline Werder Bremen & 27 & 24,3 & 130850 & 4846 & 2 & 8 & -6 \\
\hline FC Schalke 04 & 28 & 25,8 & 133700 & 4775 & 3 & 7 & -4 \\
\hline Bayer 04 Leverkusen & 26 & 24,0 & 97850 & 3763 & 4 & 5 & -1 \\
\hline Hamburger SV & 28 & 23,8 & 94750 & 3384 & 5 & 4 & I \\
\hline VfB Stuttgart & 28 & 24,8 & 94700 & 3382 & 6 & 10 & -4 \\
\hline Borussia Dortmund & 27 & 25,9 & 82750 & 3065 & 7 & 6 & I \\
\hline VfL Wolfsburg & 32 & 25,5 & 92600 & 2894 & 8 & 9 & -1 \\
\hline Hannover 96 & 27 & 26,4 & 66200 & 2452 & 9 & 13 & -4 \\
\hline Hertha BSC & 28 & 23,9 & 64100 & 2289 & 10 & 3 & 7 \\
\hline Eintracht Frankfurt & 31 & 25,1 & 62550 & 2018 & II & 12 & -1 \\
\hline VfL Bochum & 29 & 27,1 & 44350 & 1529 & 12 & 17 & -5 \\
\hline Karlsruher SC & 23 & 26,2 & 33200 & I 443 & 13 & 15 & -2 \\
\hline I. FC Köln & 29 & 26,1 & 36500 & 1259 & 14 & II & 3 \\
\hline Borussia M'gladbach & 28 & 26,7 & 34550 & 1234 & 15 & 18 & -3 \\
\hline TSG I899 Hoffenheim & 26 & 23,9 & 31950 & 1229 & 16 & I & 15 \\
\hline Arminia Bielefeld & 27 & 25,9 & 29700 & 1100 & 17 & 14 & 3 \\
\hline FC Energie Cottbus & 32 & 27,1 & 29000 & 906 & 18 & 16 & 2 \\
\hline Gesamt & 499 & 25,5 & I $4 \mid 2200$ & 2830 & & & \\
\hline
\end{tabular}

Bundesliga 2008/09 - Korrelation zur Platzierung nach der ersten Saisonhälfte

\begin{tabular}{lcccc}
\hline & Kader & $\varnothing$-Alter & Marktwert & $\varnothing$-Mw. \\
\hline Korrelation & $-0,42828$ & 0,72136 & 0,56037 & 0,56244 \\
Signifikanz & 0,03759 & 0,99947 & 0,99158 & 0,99179 \\
\hline
\end{tabular}

Natürlich stellt sich die Frage, ob die Saison 2007/08 typisch war oder eben nicht. Die Analyse sämtlicher Bundesliga-Jahrgänge seit 1963/64 muss an dieser Stelle leider entfallen. Vergleichsweise wollen wir aber noch einen Blick auf die laufende Saison 2008/09 werfen. Die entsprechenden Daten stehen in Tabelle 3, wobei sich die Platzierung auf das Ergebnis nach 17 Spieltagen bezieht, also nach der ersten Saisonhälfte. Die zugehörigen Korrelationen und (wiederum durch approximativen Permutationstest geschätzten) Signikanzniveaus sind Tabelle 3 zu entnehmen. Der größte Unterschied liegt darin, dass sich für die Kadergröße eine zu etwa 96,2\% signifikante Anti-Korrelation ergibt. Dies würde suggerieren, dass ein kleinerer Kader potenziell schlagkräftiger wäre. Die Korrelation zwischen durchschnittlichem Wert der Spieler und dem Abschneiden fällt zwar schwächer aus als in der vorigen Saison, ist mit knapp 99,18\% aber immer noch ausgeprägt.

\section{Versuch einer Interpretation}

Mindestens in den beiden betrachteten Szenarien ist die Korrelation zwischen dem Durchschnittswert der Spieler einer Mannschaft und dem Abschneiden am Ende auffällig hoch. Korrelation und Kausalität sind zweierlei, aber der Einfluss des Geldes ist hier sicherlich keine Überraschung. Interessanter finde ich die außergewöhnlich hohe Signifikanz. Diese lässt vielleicht den Schluss zu, dass die teilweise astronomischen Transfersummen gerechtfertigt sind und mindestens im Mittel den wirklichen Wert der Spieler präzise wiedergeben. Die etwas geringere Signifikanz für die erste Hälfte der laufenden Saison mag damit zusammenhängen, dass sich die wahre Stärke einer Mannschaft erst in einem langen Turnier mit vielen Spielen zeigt. Dennoch ist natürlich klar, dass das Geld allein doch keine Tore schießen kann. Eine Mannschaft aus elf superteuren Mittelstürmern wird die Meisterschaft vermutlich auch nicht holen. Es bedarf zusätzlichen Sachverstands, um die Investitionen auch in Ergebnisse umzusetzen. Und hier wiederum lohnt ein Blick auf die jeweils letzte Spalte in den Tabellen I und 3. Je höher der Eintrag desto eher scheinen Trainer und Management ihre Sache im Griff zu haben - wie zum Beispiel Felix Magath in Wolfsburg (2007/08) und Ralf Rangnick in Hoffenheim (2008/09). Umgekehrt ergibt sich eine gewisse Rechtfertigung für die eingangs erwähnten Trainerentlassungen.

Wer jetzt immer noch fragt, warum eine schöne Nebensache wie Fußball einer so ausführlichen mathematischen Betrachtung bedarf, dem sei ein Auspruch des legendären Trainers des FC Liverpool, Bill Shankly, ans Herz gelegt: "Einige Leute halten Fußball für einen Kampf um Leben und Tod. Ich mag diese Einstellung nicht. Ich versichere Ihnen, dass es viel ernster ist!"

Prof. Dr. Michael Joswig, Fachbereich Mathematik, Optimierung, TU Darmstadt, Schlossgartenstraße 7, 64289 Darmstadt joswig@mathematik.tu-darmstadt.de 